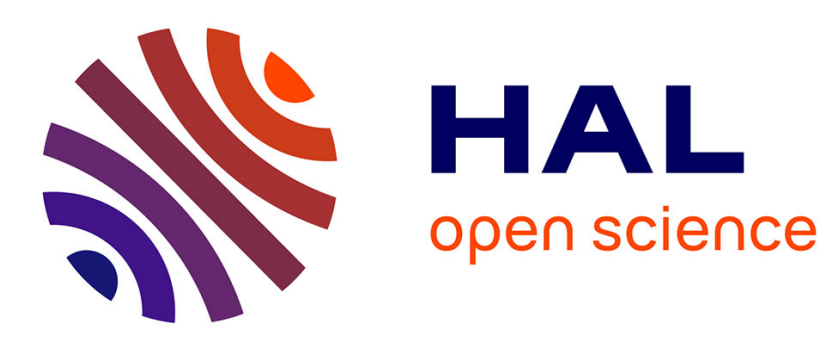

\title{
Un document arabe de la région thébaine. Réédition de P. Liebrenz Quittung
}

Naïm Vanthieghem

\section{To cite this version:}

Naïm Vanthieghem. Un document arabe de la région thébaine. Réédition de P. Liebrenz Quittung. Chronique d'Egypte; bulletin periodique de la Fondation egyptologique reine Elisabeth, 2019, 94 (187), pp.206-212. 10.1484/J.CDE.5.119070 . halshs-02974761

\section{HAL Id: halshs-02974761 https://shs.hal.science/halshs-02974761}

Submitted on 28 Dec 2020

HAL is a multi-disciplinary open access archive for the deposit and dissemination of scientific research documents, whether they are published or not. The documents may come from teaching and research institutions in France or abroad, or from public or private research centers.
L'archive ouverte pluridisciplinaire HAL, est destinée au dépôt et à la diffusion de documents scientifiques de niveau recherche, publiés ou non, émanant des établissements d'enseignement et de recherche français ou étrangers, des laboratoires publics ou privés. 


\section{Un document arabe de la région thébaine Réédition de P. Liebrenz Quittung}

Il y a maintenant quelques années, B. Liebrenz publiait une quittance arabe dont il situait la rédaction aux $\mathrm{VII}^{\mathrm{e}}$ ou au VIII ${ }^{\mathrm{e}}$ siècle $\left(^{1}\right)$. L'intérêt majeur du document résidait, pour son éditeur, dans le fait qu'il s'agit de l'un des rares documents arabes dont la provenance est assurément thébaine $\left(^{2}\right)$. B. Liebrenz y voyait un reçu émis en faveur d'un certain Šanūda, habitant de Djême, qui avait acheté la maison de sa voisine, une certaine Šağara, dont les biens étaient revenus au calife, cette dernière n'ayant pas, avant son décès, désigné d'héritier(s) $\left(^{3}\right)$. Le texte tel qu'il a été édité comporte plusieurs problèmes de lecture, qui ont conduit à une interprétation erronée de certains des éléments rapportés dans la quittance. Je propose dans cet article une réédition et une traduction du document ; elles seront suivies de considérations prosopographiques sur les personnages mentionnés.

\section{RÉÉDITION DE P. LIEBRENZ QUITTUNG}

La dénommée Daqusțas est morte sans héritier : ses biens sont donc revenus, comme c'est la coutume, au calife, c'est-à-dire à l'état $\left({ }^{4}\right)$. Son voisin Bisanda Šanūda, dont la propriété jouxte celle de Daqustas sur le flanc est, s'est porté acquéreur de la part que Daqusțas possèdait dans la maison qu'elle habitait, et ce pour la somme de trois dīnārs. Pour conclure la transaction, il a dû prendre contact avec la personne, qui avait en charge la gestion des biens revenus à l'état, le dénommé Šağara b. Abū Yazīd, qui a agi en cette affaire par l'intermédiaire

(1) B. LIEBRENZ, «Eine frühe arabische Quittung aus Oberägypten », ArchPF 56 (2010), pp. 294-314.

(2) Le seul autre document arabe de provenance thébaine - bien que n'ayant pas été rédigé sur place - est le sauf-conduit P. Ragib Sauf-conduits 3.

(3) B. LiebrenZ, «Eine frühe arabische Quittung ...» [n. 1], p. 296 : «Es handelt sich um eine Quittung in der Form einer barā'a (Freistellung), ausgestellt für einen Kopten namens Šanūda aus Šìma/Djême in der Pagarchie Armant/Hermont über drei Dinare zum Kauf eines Anteils an einem Haus aus der Erbschaft einer verstorbenen arabischen Frau namens Šağara ».

(4) Pour un cas similaire dans la documentation, voir le document édité dans A. DELATTRE \& N. VAnthieghem, « Nouveaux fragments d'un rouleau liturgique grec de l'époque fatimide », Études coptes XV. Dix-septième journée d'études coptes (Lisbonne, 18-20 juin 2015), (Paris, 2018), pp. 175-195, en part. pp. 188-192.

Chronique d'Égypte XCIV (2019), fasc. 187 - doi: 10.1484/J.CDE.5.119070 
d'un certain Qūmis, fils de Kā'il. Avant la formule de datation, la transaction est confirmée par trois témoins. Le premier, Yuhannis b. 'Abd Allāh, est, à en juger par son nom, le fils d'un chrétien récemment converti à l'islam. Le second, un certain $\mathrm{Sa}^{e} \overline{1} \mathrm{~d}$ b. Sa ${ }^{e}$, semble avoir été un musulman de naissance. Quant au troisième, Yuhannis Filūṭus, il s'agissait manifestement d'un chrétien, qui aurait même été, si l'identification que je propose est correcte, donné par ses parents pour servir au monastère de Phoibammôn. Le dernier, enfin, est un scribe chrétien - peut-être celui-là même qui a rédigé la quittance - du nom de Qūmis.

P. Halle Inv. DMG 3

Djême

$23,5 \times 22,6 \mathrm{~cm}$

vers 750

Coupon de papyrus de couleur foncée dont toutes les marges sont conservées. La marge supérieure ainsi que la marge de gauche et de droite sont étroites tandis que la marge inférieure est ample, ce qui laisse penser que le document était peut-être scellé à l'origine $\left({ }^{5}\right)$. Le texte, qui comporte dix-huit lignes, court perpendiculairement au sens des fibres. L'écriture, tracée au moyen d'un calame assez fin et à l'encre noire, est ample et élégante, même si le scribe ne parait pas des plus expérimentés; les lettres ne sont pourvues d'aucun point diacritique. Tout montre qu'un seul scribe a rédigé l'intégralité du document, les témoignages compris, ce qui est courant pour l'époque.

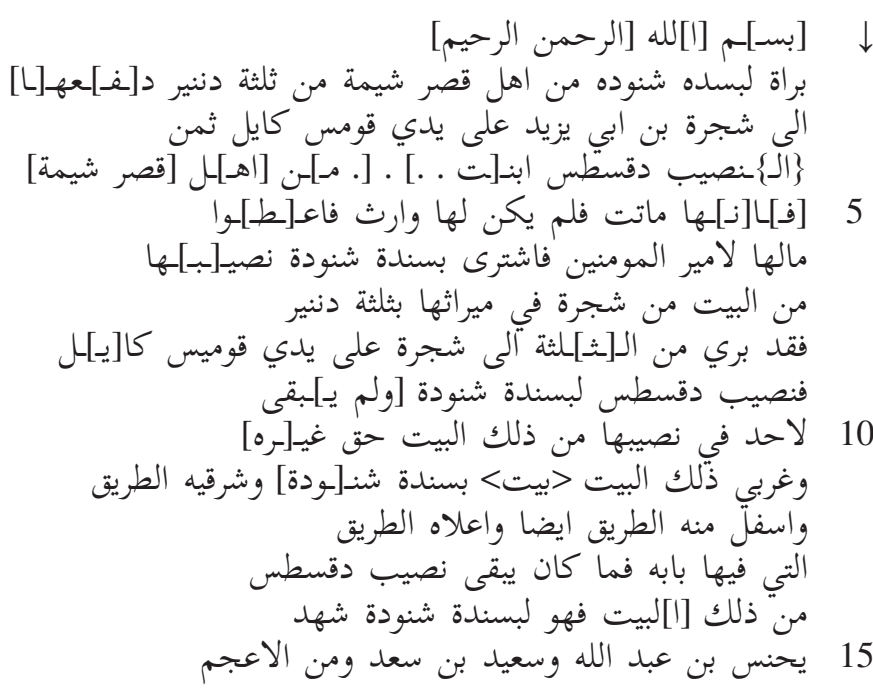

(5) Les deux fragments au bas du document semblent être rapportés et n'avoir aucun rapport avec la quittance. Celui de droite semble comporter la mention $[\tau \tilde{\eta}] \varsigma \chi \rho \varepsilon i ́ \alpha[\varsigma]$. 


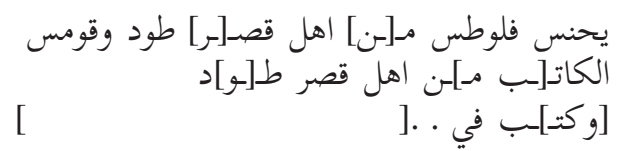

2 barā’a li-Bisanda Šanūda : barā’a li-siyāda Šanūda ed. pr. 3 'ilā Šağara b. Abì Yazīd : Šağara mar'at Yazìd ed. pr., 'alā yaday Qümis Kä̉il : 'alà yaday Qümis kämil ed. pr. 4 al-nașîb corr. ex al-bayt, Daqustas ibna[t . .] . [. m]in [ah]l [Qașr Šima] : Daqusțas išta[r]ā[-hu ...] ed. pr. 5 [fa]¿i[nna]-hā : 'i[nna]-hā ed. pr. 6 fa-štarā Bisanda Šanūda: fa-štarā li-siyāda Šanūda ed. pr. 8 Qümis Kä̉il : Qümis kāmil ed. pr. 9 [wa-lam ya]bqā : [wa-lā yabqā]] ed. pr. 11 wa-gararbi dălik al-bayt <bayt> Bisanda Šan[ūda] : wa-ġarbì dālik al-bayt <bayt> siyāda Šan[üda] $\mathbf{1 4}$ fa-huwa li-Bisanda Šanūda : fa-huwa li-siyāda Šanūda ed. pr. 15 Yuhannis b. 'Abd Allāh : Hasan b. 'Abd Allāh ed. pr. 16-17 wa-Qümis | al-kāti[b mi]n 'ahl \{Qaș\} Qạșr [Š̈ma] : wa-Qūmis I [.]l-k[... mi] $n$ 'ahl [Qaṣr? ] ed. pr. 18 [wa-kuti]ba fi : om. ed. pr.

« I ${ }^{1}$ Au nom de Dieu, le clément, le miséricordieux. $I^{2}$ Quittance en faveur de Bisanda, fils de Šanūda, habitant de Qașr Š̄ima, de trois dinars dont il s'est acquitté $\beta^{3}$ auprès de Šağara, fils d'Abū Yazīd, représenté par Qūmis, fils de Kā'il, au titre du prix $\left.\right|^{4}$ de la part de Daqustas, fille de ..., habitante de Qaṣr Šìma. $\left.\right|^{5}$ Elle est morte sans héritier et ses biens ont été donnés $\left.\right|^{6}$ au calife. Bisanda, fils de Šanūda a acheté sa part d'héritage [scil. celle de Daqusțas] ${ }^{7}$ sur la maison à Šağara pour le prix de trois dinars. $I^{8}$ Il a été déclaré quitte des trois dinars vis-à-vis de Šağara, représenté par Qūmis, fils de Kā̄il. $I^{9}$ La part appartient dès lors à Bisanda, fils de Šanūda et $I^{10}$ plus personne en dehors de lui n'a de droit sur sa part dans cette maison. I $^{11}$ À l'ouest de cette maison, se trouve la maison de Bisanda, fils de Šanūda ; à l'est, le chemin ; ${ }^{12}$ en contrebas, le chemin aussi et, au-dessus, le chemin $\mathrm{I}^{13}$ sur lequel donne la porte. La part de qu'avait Daqusțas ${ }^{14}$ sur cette maison revient à Bisanda, fils de Šanūda. Ont témoigné ${ }^{15}$ Yuhannis b. 'Abd Allāh et $\mathrm{Sa}^{e} \mathrm{~d}$ b. Sa ${ }^{e} \mathrm{i} d$, et, au nombre des musulmans, $\left.\right|^{16}$ Yuhannis b. Filuțūs, habitant de Qașr Ṭ̂ud, et Qūmis, $\left.\right|^{17}$ le scribe, habitant de Qașr Ṭūd. $\left.\right|^{18}$ Écrit le ...»

2 Bisanda Šanūda L'éditeur lisait nom du destinataire de la barā’a qu'il vocalise siyādat $\breve{S} a n \bar{u} d a$; il propose de voir dans le mot siyādat un titre ecclésiastique, qui aurait été écrit de manière défective. Un examen du rasm présent sur le papyrus montre qu'il faut lire le premier élément de cette séquence سسده, où l'on peut sans difficulté reconnaître le nom nıcente (بسندة) ; pour des occurrences de ce nom dans les documents arabes, cf. Chrest. Khoury I 77, 11 ; P. Margoliouth Monneret 4 verso, 23 et P. Tillier Deux Quittances 2, 6.

3 Šağara b. Abī Yazīd ( der Frau Yazīds »), où il interprétait le nom Šağara comme un nom féminin. Šağara est cependant un nom masculin (cf. P. Khalili I 15 verso et P. Ragib Lettres 11 verso, 1$)$.

'alā yaday Qümis Kä̉il taman La fin de cette ligne a été lue 'alā yaday Qūmis kāmil taman (« zu Händen von Qūmis, (und zwar) den gesamten Preis »), mais 
le papyrus porte plus exactement la mention 'alà yaday Qūmis Kã̉il taman (« par l'intermédiaire de Qūmis, fils de Kā’il, au titre du prix »). L'orthographe $K \bar{a} i l$ est une variante pour Kîl, la transcription usuelle du nom copte Chael. ipation, le scribe semble avoir écrit dans un premier temps al-bayt avant de se دقسطس

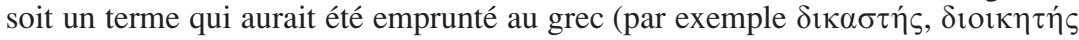

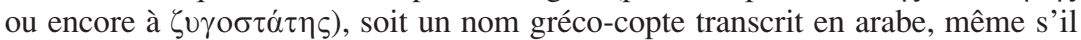
ne sait de quel nom il s'agit - il suggère ткшстос, le pendant féminin de кшстос, ou le nom romain T $\varepsilon \chi \varepsilon \sigma \delta \varepsilon \cup \varsigma / T \varepsilon \chi \varepsilon \sigma \tau \varepsilon \cup \varsigma$. La solution est beaucoup plus simple : il s'agit d'un nom féminin constitué de la préformante Td- (« celle de ») et du nom gréco-latin Costas (en copte кшста), qui est lui-même une variante du nom Constans ; sur cette formation de noms, voir L. REINFANDT \& N. Vanthieghem, "Les archives fiscales de Mīnā, fils de Damarqūra, un contribuable copte du $\mathrm{IX}^{\mathrm{e}}$ siècle », Mélanges Jean Gascou. Textes et études papyrologiques (P. Gascou), pp.351-370, en part. p. 354. Après le nom Daqusțas, l'éditeur proposait de lire la forme verbale išta[r] $\bar{a}[-h u$...] dans le rasm اد dans son commentaire, il envisagait la possibilité d'une lecture ibn[at]. L'espace entre les différentes dents qui suivent l'alif montre qu'il ne peut pas s'agir d'un sìn ou d'un šinn, mais bien de deux lettres différentes. Étant donné que Daqustas est une femme, seule une lecture ibn [at] paraît possible. Après le patronyme, on s'attend à ce que la quittance indique où cette femme habitait. Puisque son voisin, Bisanda, fils de Šanūda, habitait Djême, il faut nécessairement que Daqusțas, fille de ..., ait habité la même bourgade.

5 [fa]-'i[nna-]hā mätat L'éditeur lisait 'i[nna-]hā mātat, mais il manque un motlien qui fasse la transition entre ce qui précède et les nouvelles informations données. Je propose d'insérer la conjonction $f a$-.

9 [wa-lam ya]bqā L'éditeur n'a pas remarqué la présence de la séquence ]bqāa que l'on lit sur un fragment mal replacé par le restaurateur. B. Liebrenz lisait en outre [ $w a-l \bar{a}$ yabqā], mais on attend habituellement dans les documents juridiques une forme d'apocopée wa-lam yaqba (cf. P. Cair. Arab. 114, 6 et P. Fahmi Taaqud 5, 13), parfois écrite sous une forme fautive wa-lam yabqā (cf. CPR XXVI 38, 4 et P. David-Weill Louvre 20, 10).

15 Yuhannis b. 'Abd Allāh B. Liebrenz lisait le nom du premier témoin Ḥasan b. 'Abd Allāh, mais le papyrus porte en réalité le nom bien plus intéressant Yuhannis b. 'Abd Allāh. Ceci implique que ce personnage n'était pas un simple musulman, mais qu'il s'agissait d'un converti, ou à tout le moins du fils d'un converti.

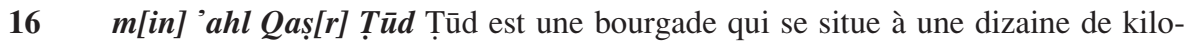
mètres au sud de l'actuelle ville de Louxor, juste en face de la ville d'Armant. Sur ce toponyme, voir É. AmÉLINEAU, La géographie de l'Égypte à l'époque copte (Paris, 1893), pp. 520-521 ainsi que S. TIMM, Das christlich-koptische Ägypten in arabischer Zeit (Wiesbaden, 1984-1992), pp. 2862-2865.

16-17 Qümis I al-kät[ib mi]n ahl Qaṣr T[ü]d L'éditeur lisait le premier élément et ne déchiffrait que des bribes de ce qui suivait. On reconnaît sans trop de difficulté le mot $a l-k a \bar{t} t i b$ - qui pourrait indiquer que Qūmis a agi comme scribe de la quittance. Quant à la provenance du personnage, les traces qui subsistent montrent qu'il était originaire de Qașr Tūd comme le témoin précédent. 


\section{PROSOPOGRAPHIE DES PERSONNAGES MENTIONNÉS}

La quittance mentionne une série de personnages qui n’ont pas été identifiés dans l'editio princeps. L. Berkes, dans son livre sur l'organisation administrative des villages, a cependant ouvert la voie en proposant, à juste titre, de reconnaître en Qūmis, fils de Kā’il, le dioikêtês Komês, fils de Chael, dont la période d'activité se situe entre 748 et $759\left(^{6}\right)$. L'identité de la plupart des autres personnages qui apparaissent dans le document peut aussi être précisément établie. Le premier, Bisanda, fils de Šanūda, en faveur duquel la quittance est émise, fut manifestement un notable de la région thébaine $\left(^{7}\right)$. Dans le testament $P . K R U$ 70 , un certain Pisente et son père, l'higoumène Chenouté $\left(^{8}\right)$, sont en effet mentionnés aux nombres des héritiers d'une certaine, Tbastes, fille d'Apa Biktôr et de Thrabonia, une femme sans enfant qui voyant sa fin approcher rédige un testament le 4 juillet 750. Dans $P$. KRU 20, le même homme achète, en 759, une partie de maison à un certain Stephanos, fils de Germanos.

Un autre personnage qui mérite sans doute plus d'attention que celle qu'on lui a portée est le premier témoin chrétien de la quittance, un certain Yuhannis Filūtus. Ce dernier doit vraisemblablement être confondu avec Johannes, fils de Philotheos, petit-fils de Chenouté qui, d'après $P$. KRU 90, fut donné par ses parents pour servir au monastère de Phoibammôn $\left({ }^{9}\right)$. La fréquence du nom et du patronyme interdit cependant toute certitude.

Quant à Qūmis, le scribe, originaire de Tūd, on pourrait prudemment suggérer de l'identifier avec l'homonyme, fils de Mariam et de Daniel, originaire de Taut, qui fut lui aussi oblat au monastère de Phoibammôn dans le deuxième quart du VIII ${ }^{\mathrm{e}}$ siècle $\left({ }^{10}\right)$.

Reste à comprendre qui est le musulman Šağara b. Abī Yazīd à qui les trois dinars sont versés. Il s'agit nécessairement d'un haut fonctionnaire de l'administration arabe puisqu'il a en charge la gestion des héritages revenus à l'état à la suite du décès de personnes qui n'avaient pas d'héritiers. Šağara pourrait-il avoir été pagarque de l'Hermonthite? Je crois pouvoir reconnaître son nom et son patronyme, sous une forme trompeuse dans le prescrit du testament

(6) Sur la datation de ce texte, voir L. BERKES, Dorfverwaltung und Dorfgemeinschaft in Ägypten von Diokletian zu den Abbasiden (Wiesbaden, 2017), pp. 245-246.

(7) W. TILL, Datierung und Prosopographie der koptischen Urkunden aus Theben (Vienne, 1962), p. 167.

(8) W. TILl, Datierung und Prosopographie [n. 7], p. 210.

(9) W. TILl, Datierung und Prosopographie [n. 7], p. 109.

(10) W. TILl, Datierung und Prosopographie [n. 7], p. 122. W. Till proposait une datation au milieu du VIII ${ }^{\mathrm{e}}$ siècle, mais le document ayant été écrit par le scribe Aristophanês, dont on connaît bien la période d'activité (voir J. CROMwELl, Recording Village Life : A Coptic Scribe in Early Islamic Egypt [Ann Arbor, 2017], en part. p. 78), il faut plus placer la rédaction du contrat quelque part entre janvier 724 et novembre 756 . 
P. KRU 70 (4 juillet 750), dont L. Berkes et J. Cromwell ont récemment revu

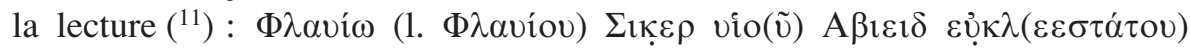

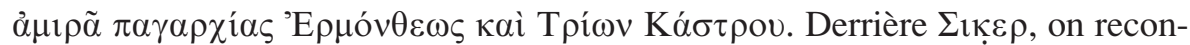
naît sans trop de peine le nom Šağara, sachant que le son /š/ est parfois rendu en grec au moyen d'un sigma, que le son /ğ/ peut être transcrit par un kappa $\left({ }^{12}\right)$; il faut juste admettre que le dernier /a/ n'est pas noté. Quant au patronyme $\mathrm{A} \beta \imath \varepsilon \imath \delta$, on peut y voir simplement une faute pour la kunyā $\mathrm{A} \beta 1 \varepsilon \zeta \imath \delta$, dont le zêta aurait été omis $\left({ }^{13}\right)$. Cette identification apparaît d'autant plus probable à mes yeux que le testament $P$. KRU 70 déjà mentionné fait justement intervenir à la fois le dioikêtês Komês, fils de Chael en même temps que Pesynte et son père Chenouté.

LA PRÉSENCE ARABE DANS LA RÉGION THÉBAINE AU DÉBUT DE L'ÉPOQUE ABBASSIDE

Cette quittance témoigne en définitive de l'implication croissante des pagarques arabes - ici à travers un subordonné -, donc de l'état omeyyade puis abbasside, dans la gestion quotidienne d'une province d'Égypte aussi lointaine que la pagarchie d'Hermonthis. Ce n'est sans doute pas tant au titre de ses pouvoirs judiciaires que Šağara $b$. Abī Yazīd se saisit dans notre document $\left({ }^{14}\right)$, tout comme d'ailleurs dans le testament $P$. KRU 70, du cas d'une femme sans enfants, qu'au titre de ses prérogatives fiscales : l'absence d'héritier direct - ascendant ou descendant - impliquait en effet que la fortune de la défunte revienne au fisc arabe $\left({ }^{15}\right)$. Paradoxalement, alors que la présence de l'état arabe se fait davantage tangible dans la région thébaine, le nombre de musulmans résidant dans la pagarchie reste vraisemblablement assez limité. La quittance semble ainsi rédigée par une personne - peut-être le scribe Qūmis mentionné au nombre des témoins -, qui, bien que n'étant pas illettrée, n'avait manifestement ni l'expérience ni la science des scribes contemporains que l'on connaît pour le Fayoum

(11) L. Berkes \& J. Cromwell, « An amīr between Umayyads and Abbasids: A Note on P. KRU 70 », CdÉ 93 (2018), pp. 218-220.

(12) Sur le rendu des phonèmes arabes en grec, voir A. KAPLONY, « On the Orthography and Pronunciation of Arabic Names and Terms in the Greek Petra, Nessana, Qurra, and Senouthios Letters (Sixth to Eighth Centuries CE) », Mediterranean Language Review 22 (2015) pp. 1-81, en part. pp. 1-12 ainsi que A. AL-JALLAD, « The Arabic of the Islamic Conquests: Notes on Phonology and Morphology based on the Greek Transcriptions from the first Islamic Century », BSOAS 80 (2017), pp. 419-439.

(13) L. Berkes et J. Cromwell, après avoir pris conseil auprès d'arabisants (dont moi), proposaient prudemment de reconnaître le nom Šukayr b. 'Ubayd, mais il me semble qu'il faut à présent renoncer à cette interprétation.

(14) Sur ces prérogatives, voir à présent M. TILliER, L'invention du cadi. La justice des musulmans, des juifs, des chrétiens aux premiers temps de l'Islam (Paris, 2017), pp. 50-77.

(15) Sur cette pratique, voir entre autres H. RABIE, The Financial System of Egypt A.H. 564741/A.D. 1169-1341 (Oxford, 1972), pp. 127-130. 
ou l'Hermopolite. Ceci m'invite à penser que l'administration locale ne disposait pas, contrairement à des pagarchies septentrionales, d'un grand nombre de gens capables d'écrire l'arabe - d'où peut-être aussi le nombre limité de documents arabes que nous connaissons pour la région thébaine. C'est sans doute aussi le nombre réduit de musulmans dans la région qui explique que l'on ait recouru à deux témoins chrétiens pour attester de la validité de la transaction alors que le témoignage de non-musulmans n'est pas chose usuelle dans les documents juridiques. Le cas du premier témoin, originaire de Djemé, le dénommé Yuhannis b. ‘Abd Allāh, dont je pense pouvoir dire qu'il était le fils d'un chrétien récemment converti, suggère enfin peut-être qu'au début de l'époque abbasside la bourgade et sa région commençaient seulement à connaître ses premières conversions alors que le mouvement s'était amorcé plus tôt dans d'autres districts égyptiens. 